\title{
LICENSE PLATE RECOGNITION OF MOVING VEHICLE
}

\author{
A. SHAWKY ${ }^{1}$, A. HAMDY ${ }^{2}, H . K E S H K^{2}$, and M. EL_ADAWY ${ }^{2}$ \\ ${ }^{1}$ Teaching Assistant, Modern Sciences and Art University (MSA), Egypt \\ ${ }^{2}$ Comm. And Electronics Dept., Faculty of Engineering, Helwan University, \\ Cairo, Egypt
}

(Received July 21, 2009 Accepted September 14, 2009).

\begin{abstract}
This paper presents a license plate recognition system of moving vehicles for Egyptian license plates. The proposed system extracts the characters features of a plate from a captured image by a digital video camera. Then it identifies numbers and characters of the plate using a multilayer neural network. The proposed system consists mainly of two processes: the first one is the training process, in which a database is build and the neural networks used for recognition are trained. The second one is the recognition process which consists of vehicle detection from the stream of frames, a plate position estimation, words and numbers segmentation, and plate recognition stages.
\end{abstract}

KEYWORDS: License Plate Recognition (LPR), Vehicle Detection, Optical Character Recognition, Plate Localization, Plate Segmentation.

\section{INTRODUCTION}

License plate recognition (LPR) is a good tool for automatic vehicle and traffic monitoring using digital image processing. For implementing an LPR system, digital image processing techniques and an artificial neural network can be used. These applications provide access entering car parks, register (specification and time) [1, 2], depots and restricted areas which is necessary for security [3]. It can also be applied to automating fuel supplies in filling station and traffic law enforcement $[4,5]$, congestion pricing [6], traffic control management for recognizing vehicles that commit traffic violation, such as: entering restricted area without permission, breaking speed limits; etc.

Most previous applications and researches have in some way restricted their working conditions [7], such as limiting them to indoor scenes, stationary backgrounds [8], fixed illumination [4], fixed type of license plate, prescribed driveways [9, 10], limited vehicle speeds [11], or designated ranges of the distance between camera and vehicle [12].

The aim of this application is to remove some of these restrictions. Of the various working conditions, outdoor scenes and no stationary backgrounds may be the two factors that most influence the quality of scene images acquired and in turn the complexity of the techniques needed.

Egyptian licenses plate format have different background colors (Taxi background color is orange) while private license back ground color is white as shown in Fig.1, which complicates this application. All types use a set of digits and words in Arabic. 
Therefore, an optical character recognition (OCR) for identifying numbers and words is needed.

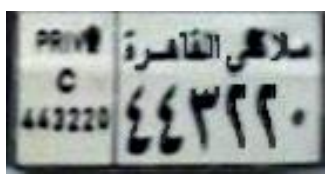

(a)

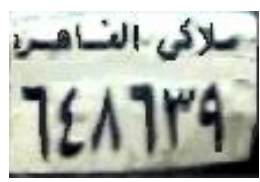

(b)

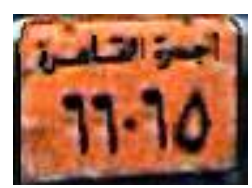

(c)

Fig.1.Different Egyptian license plates

\section{THE PROPOSED LPR ALGORITHM}

In such an algorithm, one of the most difficult tasks is to detect the vehicle from captured images then locate the license plate, which could be anywhere in the image. The following assumptions about the plates considered in this algorithm are made:

1- The plates have a rectangular shape with two rows of characters.

2- The plate has dark characters on a bright white or a bright orange background.

3- The orientation of the license plate is nearly aligned with the vertical and horizontal axes.

This algorithm works with both color and gray video which is cut to frames consisting of $400 * 300$ pixel transferred to computer memory. Our system consists of four stages: vehicle detection, number plate localization stage, segmentation stage and the recognition stage which consists of two processes: The training and testing processes.

\section{VEHICLE DETECTION}

In this step, the recorded video is divided into frames and stored on computer memory. The system checks them one by one. When a vehicle is detected, the frame is sent for processing in the next stage.

As an initial way of eliminating frames that do not contain a moving vehicle is to take the background frame and subtract any new frame from it. If this value of subtraction is within certain limit, no new frame (no vehicle on this frame) is considered. To speed up calculations the Stauffer-Grimson algorithm [13] was used. The alteration was to just divide the frame into small areas; each area contains four pixels in both the horizontal and the vertical dimension [14]. A single pixel decides for an area of sixteen pixels whether it should be classified as background or foreground. For a single area, instead of making sixteen comparisons between the new frame and the background frame, the system make one comparison to the selected pixel, reducing computational cost by a factor of sixteen.

In our algorithm, the window is increased to $10 * 10$ in order to reduce the computational cost by a factor of one hundred. The background can be calculated as the average of the first 100 frames from the captured video. This background can be calculated from time to time to avoid changing of illumination during daytime. 


\section{PLATE LOCALIZATION STAGE}

To locate the plate license number, follow the algorithm shown in Fig.2.



Fig.2. Flowchart to the plate localization algorithm

\subsection{Finding the Horizontal Position of the Plate}

For each horizontal line at the binary frame, the number of changes to pixels color from black to white is counted. For example Fig.3 counts all horizontal lines changes.
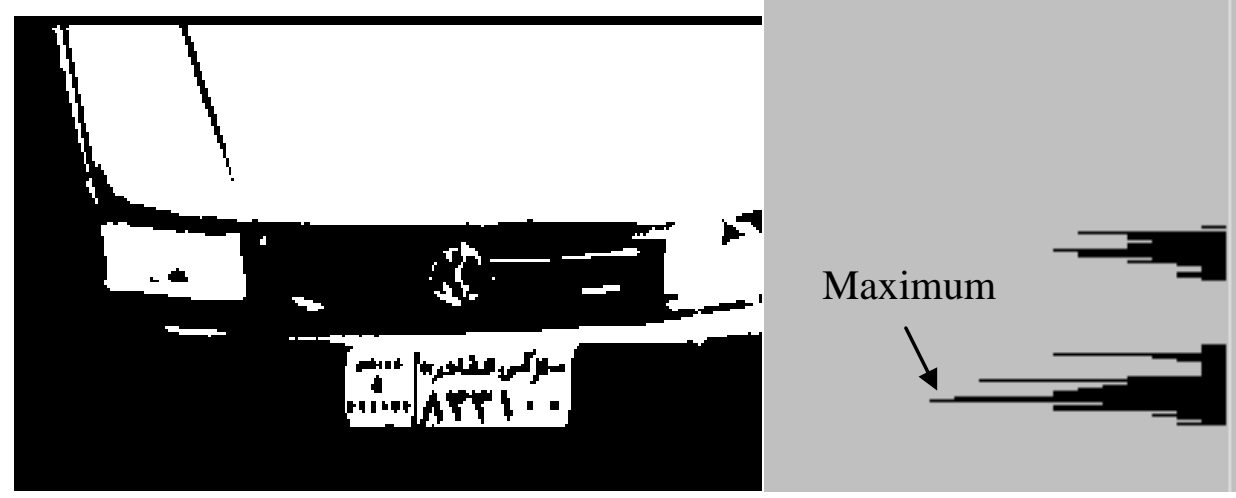

Total number of changes

Fig.3.The number of changes from black to white 
At the plate area, the maximum number of changes to pixels color from black to white is located. To have the accurate plate area, the average changes of three consecutive lines to find maximum value was taken, as shown in Fig.4. This area led us to the horizontal location of the plate as shown in Fig .5.
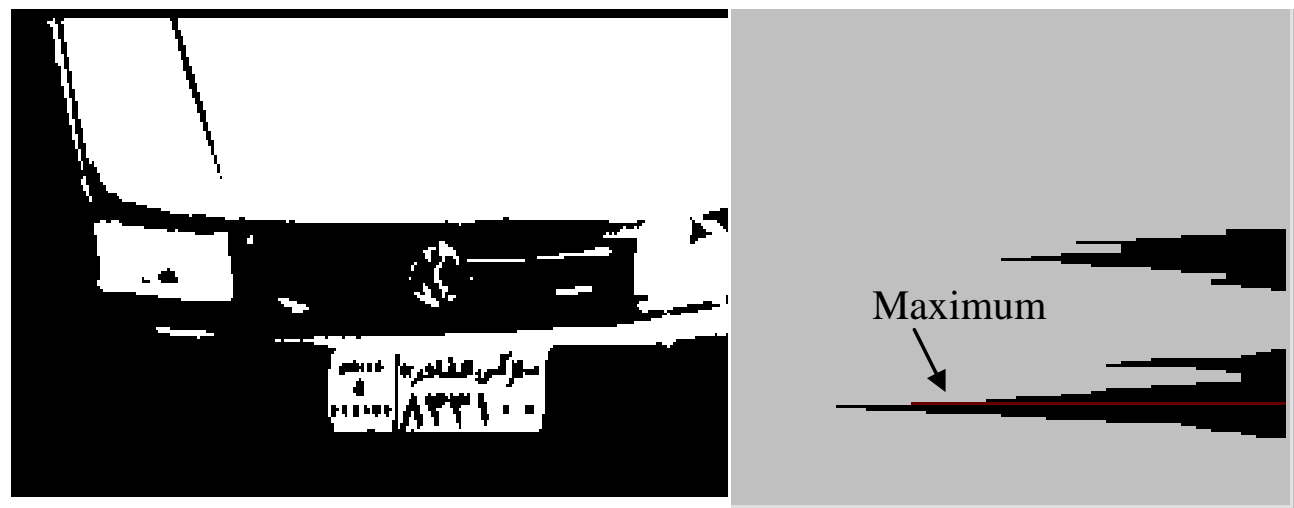

Total number of changes

Fig. 4. The average changes of three consecutive lines

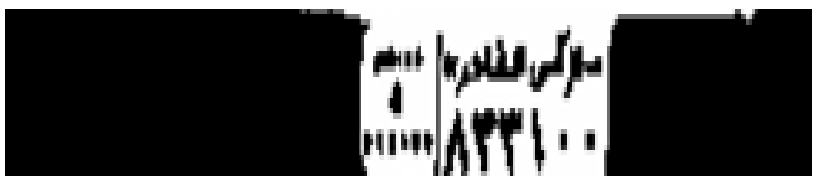

Fig.5. Horizontal window of the plate

\subsection{Vertical Edges Detection on the Horizontal Window of the Plate}

There are many edge detector filters. Most of them work on the assumption that an edge occurs where there is discontinuity in the intensity function. Using this assumption, if the derivative of the intensity values is taken across the frame and the point where derivative is a maximum is found, we will have marked out edges. To detect the edges separately, Prewitt mask [15] on vertical direction was used as shown in Fig.6.

\subsection{Finding the Left and Right Edges of the Plate}

There are many vertical edges on the horizontal window of the plate. By studying the width of the plate according to image width, one can notice that the plate width is greater than the quarter of frame width. If the frame is divided into four equal areas; we should find only one plate edge at most in each area. Hence, the two edges in two successive windows will represent the left and right edges of the plate as shown in Fig.6. 




(b)

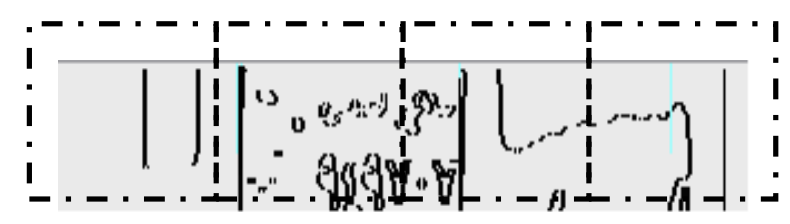

(c)

Fig.6. Finding the left and right edges of the plate

a) Horizontal window of the plate

b) Prewitt mask on vertical direction

c) The left and right Edges of the plate

\section{SEGMENTATION}

The next step after the identification of the rectangular plate is to isolate its words or digital contents. All plates are assumed to contain two rows of words and digital contents.

\subsection{Isolating between Words and Digital Contents}

We need to isolate two regions as shown in Fig.7.
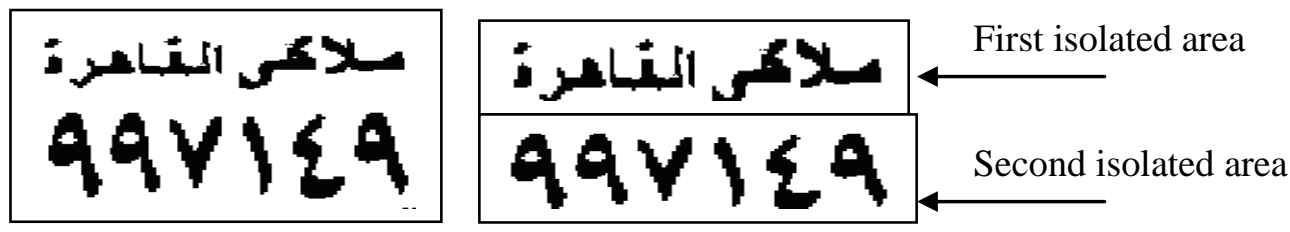

Fig.7.The extracted plate and required isolation

To do this, the horizontal summation of all black pixels in each row is calculated. The peaks of the summation will identify the two rows of characters as shown in Fig.8. 



No. of Pixels

Fig.8. Horizontal projection to isolate plate a) The plate, b) Horizontal projection to the plate

\subsection{Vertical Projection for Numeric Row}

By counting the number of black pixels in each column (vertically projection), the peaks of digits will represent the existence of a digit, and the valley will represent the isolation or boundary between these digits, as shown in Fig.9.

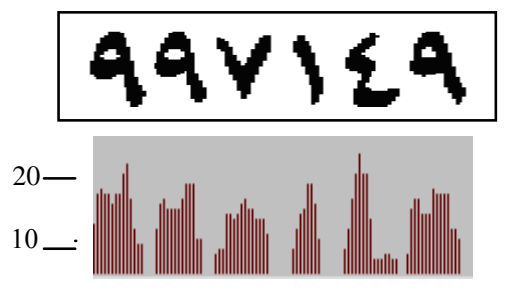

Fig.9. Vertical projection to isolate the different digits a) The extracted row, b) The vertical projection

This method of isolating digits has two advantages:-

1- Independence of character position and also independence of the size of character.

2- The number of characters in the row is not fixed, maybe five characters or six. This method suffers from some problems especially with the Egyptian license plates. For example, bolts used to fix the plate may appear as a dot that may disturb the recognition process, as shown in Fig. 10.

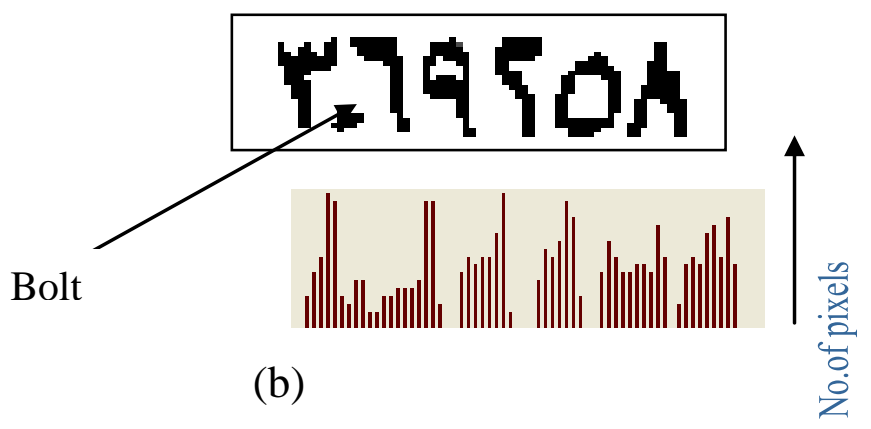

Fig.10.The problem caused by fixing bolt a) The extracted row, b) The vertical projection 
This problem is solved by putting maximum distance (width) to one digit. If the digit size is more than this limit, this digit will be divided into two digits at the valley point. The maximum distance (width) to one digit is factor of extracted plate width.

\subsection{Vertical Projection for Registration Row}

By the same way, vertical projection for the registration row is used to separate the

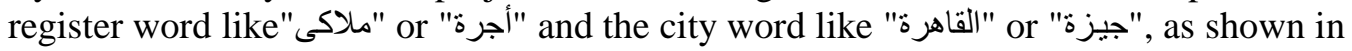
Fig. 11.

(a)

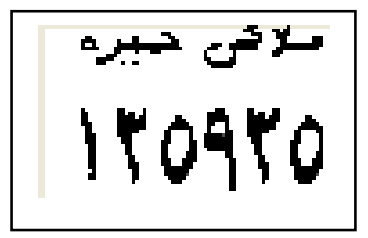

(b)

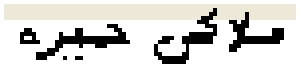

(c)
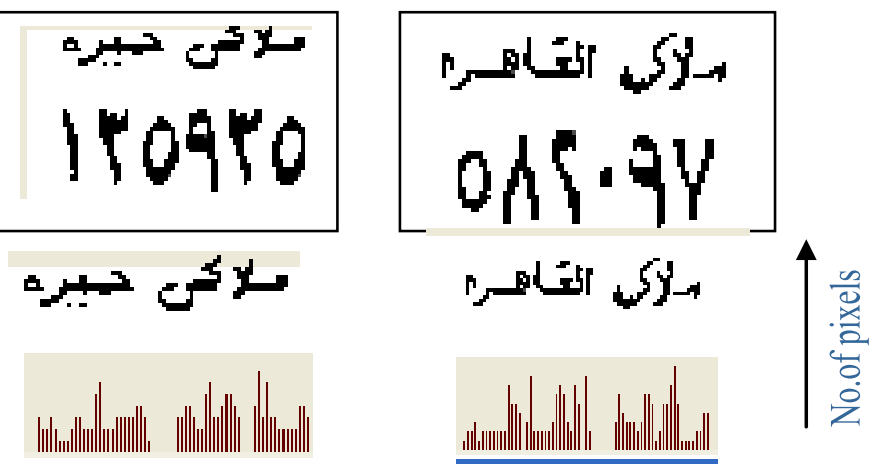

(d)
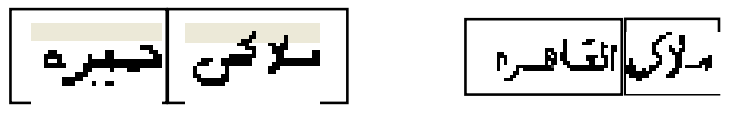

Fig.11. Vertical projection for the registration row

a) Extracted plate b) Extracted row, c) Vertical projection

d) Isolated words

\section{RECOGNITION STAGE}

Three neural networks are used to identify the following data

1. The digits from 1 to 9 .



3. Words "الجاهرة" and "الجيز" "ماهر"

Our numerical neural network has 19 input indexes. The first 12 inputs are obtained by dividing the number to 12 rectangles (R1:R12), and compute the intensity for each rectangle as shown in Fig.12. The other seven inputs are obtained by dividing the number horizontally into four regions $(\mathrm{H} 1: \mathrm{H} 4)$ and vertically into three regions (V1:V3). For example the area $\mathrm{H} 1$ consists of $\mathrm{R} 1, \mathrm{R} 2$ and $\mathrm{R} 3$, and the area $\mathrm{V} 1$ consists of R1, R4, R7 and R10. The total summation of pixel changes from white to black is computed for the seven regions, each region has one input index. 


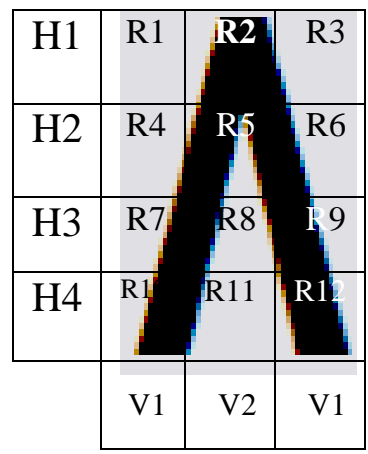

Fig.12. Numerical Neural network 19 input indexes

Each number is divided into 12 rectangles according to this formula

$$
F(i, j)=\frac{\sum \text { Num WhitePixels }(i, j)}{\sum \text { Area }(i, j)}
$$

The other seven input indexes are computed according to this formula

$$
F(i)=\frac{\sum \text { NumChangeBetweenBlakAndWhite }}{\sum \text { countNumORRowsOrColum } n}
$$

This neural network has one hidden layer with 15 neurons, which is selected by trial and error method and the number of outputs is nine.

"القاهرة" ,"ملاكى " , "اجرة" By the same way, the system can recognize the words and " الجيزة".

\section{EVALUATION RESULTS}

Two aspects were evaluated in the proposed system: the plate localization and the plate characters recognition. In both cases, the system performance was evaluated using 15 minutes recorded video for moving vehicles containing 290 vehicles, all of them corresponding to vehicles front sides. In the first case, that is the plate position estimation, a correct localization average of $96.9 \%$ was obtained. In the second case, the system ability to recognize the segmented plate characters independently was evaluated with a recognition rate of $97.6 \%$.

\section{CONCLUSIONS}

This paper presents a license plate recognition system of moving vehicle for Egyptian license plate. This system helps applications such as the assistance in the detection and identification of stolen vehicles, access control to some exclusive places, etc. The proposed system consists mainly of two processes: the training process, in which a database is built and the neural networks used for recognition are trained and also the recognition process which consists of vehicle detection from the stream of frames, a plate position estimation, segmentation, and plate recognition stages. 


\section{REFERENCES}

1. Sirithinaphong, T., and Chamnongthai, K., "The Recognition of Car License Plate for Automatic Parking System", in Proc. 5th Int. Symp. Signal Processing and its Applications, pp. 455-457, 1998.

2. Yung, N.H.C., Au, K.H., and Lai, A.H.S., "Recognition of Vehicle Registration Mark on Moving Vehicles in an Outdoor Environment", in Proc. IEEE Int. Conf. Intelligent Transportation Systems, pp. 418-422, 1999.

3. Draghici, S., "A Neural Network Based Artificial Vision System for License Plate Recognition”, Int. J.Neural Systems, Vol. 8, pp. 113-126, 1997.

4. Davies, P., Emmott, N., and Ayland, N., "License Plate Recognition Technology for Toll Violation Enforcement", Inst. Elect. Eng. Colloquium Image Analysis for Transport Applications, pp. 7/1-7/5, 1990.

5. Yamaguchi, K., Nagaya, Y., Ueda, K., Nemoto, H., and Nakagawa, M., "A Method for Identifying Specific Vehicles Using Template Matching", in Proc. IEEE Int. Conf. Intelligent Transportation Systems, pp. 8-13, 1999.

6. Cowell, J.R., "Syntactic Pattern Recognizer for Vehicle Identification Numbers", Image and Vision Comput., Vol. 13, No. 1, pp. 13-19, 1995.

7. Emiris, D.M., and Koulouriotis, D.E., "Automated Optic Recognition of Alphanumeric Content in Car License Plates in a Semi-structured Environment”, in Proc. Int. Conf. Image Processing, Vol. 3, pp. 50-53, 2001.

8. Salgado, L., Menendez, J. M., Rendon, E., and Garcia, N., "Automatic Car Plate Detection and Recognition Through Intelligent Vision Engineering", in Proc. IEEE Int. Carnahan Conf. Security Technology, pp. 71-76, 1999.

9. Miyamoto, K., Nagano, K., Tamagawa, M., Fujita, I., and Yamamoto, M., "Vehicle License Plate Recognition by Image Analysis", in Proc. Int. Conf. Industrial Electronics, Control and Instrumentation, pp. 1734-1738, 1991.

10. Parisi, R., Claudio, E.D.D., Lucarelli, G., and Orlandi, G., "Car Plate Recognition by Neural Networks and Image Processing", in Proc. IEEE Int. Symp. Circuits and Systems, Vol. 3, pp. 195-198, 1998.

11. Adorni, G., Bergenti, F., and Cagnoni, S., "Vehicle License Plate Recognition by Means of Cellular Automata", in Proc. IEEE Int. Conf. Intelligent Vehicles, pp. 689-693, 1998.

12. Naito, T., Tsukada, T., Yamada, K., Kozuka, K., and Yamamoto, S., "Robust License Plate Recognition Method for Passing Vehicles Under Outside Environment", IEEE Trans, Vol. 49, pp. 2309-2319, 2000.

13. Chris, S., "Adaptive Background Mixture Models for Real-Time Tracking", In Proc. Conf. Computer Vision and Pattern Recognition, pp. 246-252, 1999.

14. Fredrik, T., "Real Time Automatic License Plate Recognition in Video Streams", Mathematical Imaging Group, Lund University, Sweden, 2007.

15. Gonzalez, R.C., and Woods, R.E., "Digital Image Processing", 2nd Edition, Prentice Hall, Upper Saddle River, 2002. 


\section{التعرف على اللوحة المعدنية لسيارة متحركة}

يصف هذا البحث نظام للتعرف على اللوحة المعنية المصرية لسيارة متحركة. والذى يقوم على تحديد

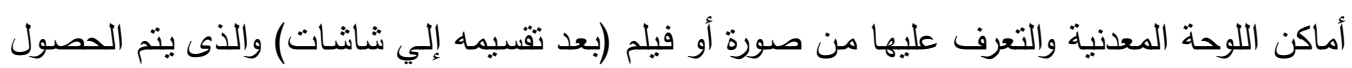

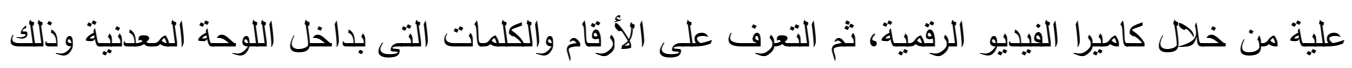
باستخدام الثبكات العصبية. والنظام المقترح يشمل نقطتين رئيسيتين : الأولى هى على علية تدريب النيام النظام

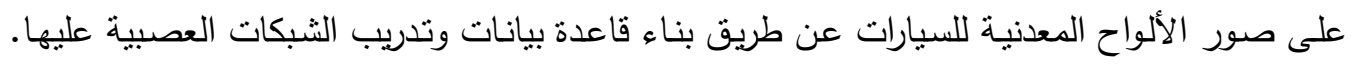

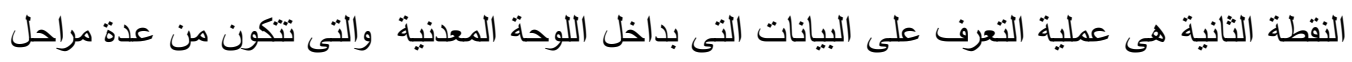
هم تحديد الثاشـة النى تحتوى على السيارة داخل الفيلم ثم تحديد مكان اللوحـة المعدنيـة، واستخراج الكلمات والأرقام والتعرف عليهم. 